\title{
Madres solteras: estigma sexista desde el imaginario cultural: caso Universidad Técnica de Manabí
}

Single mothers: sexist stigma from the cultural imaginary: case Universidad Técnica de Manabí

Gissella Andrea Mieles Anchundia ${ }^{1}$

Fabián Gustavo Menéndez Menéndez² Jacqueline Coromoto Guillen de Romero 3

RECIBIDO: 28 DE ABRIL DE 2020

ACEPTADO: 15 DE JUNIO DE 2020

\section{RESUMEN}

Las madres solteras del siglo XXI siguen siendo víctimas del orden social y patriarcal, el estigma sexista ha involucrado la reasignación de roles como parte de las prácticas del entorno social. El objetivo de la investigación es caracterizar el estado situacional de las madres solteras, referente al estigma de roles, desde la Universidad

\footnotetext{
Egresada de Trabajo Social, Facultad de Ciencias Humanísticas y Sociales, Universidad Técnica de Manabí, Portoviejo, Ecuador; gmieles6510@utm.edu.ec, ORCID: https:// orcid.org/0000-0001-6144-456X Google Scholar: https://scholar.google.es/citations?hl=es\&user=KnQPfPgAAAAJ

2 Magister en Educación y Desarrollo Social, Licenciado en Ciencias de la Comunicación Social, Docente, Universidad Técnica de Manabí, Portoviejo, Ecuador; fmenedez@utm.edu.ec, ORCID: https:// orcid.org/0000-0002-5387 Google Scholar: https://scholar.google.es/citations?hl=es\&user=Qgpc87wAAAAJ

3 Doctora en Ciencias Juridicas, Licenciada en Trabajo Social, Docente, Universidad Técnica de Manabí, Portoviejo, Ecuador; j.guillen@utm.edu.ec, ORCID: https://orcid.org/0000-0002-9112-1910

Google Scholar: https://scholar.google.es/citations?hl=es\&user=9RCEPkOAAAAJ
} 
Técnica de Manabí, Portoviejo, Ecuador. El proceso de investigación está orientado bajo un enfoque positivista, con una metodología cuantitativa, descriptiva, analítica, exploratoria, bibliográfica y estadística. Se establecen tres momentos: el estado situacional y demográfico, el examinar las edades de las mujeres participantes y la correlación de los resultados que se obtienen del procesamiento de datos. Los aportes indican que las madres solteras, que son parte de la población de estudio, inciden en que el factor económico es una de las principales desventajas de llevar el rol de crianza completa; además, que el estigma abarcado desde esta cultura se ve reflejada en el grado de desigualdades sobre la corresponsabilidad y los bienes adquisitivos dentro del sistema patriarca, que la mujer asume un rol total como parte del patriarcado y la incidencia machista. El apoyo familiar es una de las principales fuentes de sostén para las madres solteras, porque se encuentra con una sostenibilidad para poder desarrollar la actividad académica universitaria.

Palabras clave: madre soltera, familia monoparental, imaginario cultural, sexismo, roles de género

\section{ABSTRACT}

Single mothers of the 21st century continue to be victims of the social and patriarchal order, the sexist stigma has involved the reassignment of roles as part of the practices of the social environment. The objective of the research is to characterize the situational status of single mothers, referring to the stigma of roles, from the Technical University of Manabí, Portoviejo, Ecuador. The research process was oriented under a positivist approach, with a quantitative, descriptive, analytical, exploratory, bibliographic and statistical methodology. Three moments are established; the situational and demographic status, examining the ages of the participating women and the correlation of the results obtained from the data processing. The contributions indicate that single mothers who are part of the study population; Incidence that the economic factor is one of the main disadvantages of carrying out the role of complete upbringing, in addition to the stigma encompassed from this culture that is reflected in the degree of inequalities on co-responsibility and purchasing assets within the patriarch system, and that women assume a total role, as part of patriarchy and male advocacy. Family support is one of the main sources of support for single 
mothers because it meets sustainability in order to carry out university academic activity.

Keywords: single mother, single parent family, cultural imaginary, sexism, gender roles

\section{Introducción}

El 8 de marzo se conmemora el "Día Internacional de la Mujer" y la "Organización de las Naciones Unidas (ONU)" es uno de los organismos partícipes de la fecha. El mundo ha conseguido muchos avances, pero ningún país ha alcanzado la equidad de género. Hace 50 años el hombre llegó a la Luna y en la última década se ha descubierto nuevos ancestros humanos y se ha fotografiado un agujero negro por primera vez. Mientras tanto, existen restricciones en la ley que impiden a 2700 millones de mujeres contar con las mismas opciones laborales que los hombres. Menos del $25 \%$ de los parlamentarios eran mujeres en el 2019 y una de cada tres mujeres sigue sufriendo violencia de género.

Sin embargo, frente a las cifras presentadas, el desarrollo de la mujer en Latinoamérica puede enmarcar aspectos de gran estudio sociológico. En el marco de la XVI "Conferencia Regional sobre la Mujer de América Latina y El Caribe", se presentó el documento sobre la autonomía de las mujeres en escenarios económicos cambiantes (CEPAL, 2020). Una puesta de escena de este proceso es el cambio sobre el cumplimiento de autonomía que deben tener las mujeres en este siglo XXI. Desde antes, a las madres solteras se les ha considerado parte de una sociedad que no encaja; "en la antigüedad e incluso hasta hoy en ciertos contextos, las madres solteras son víctimas de discriminación, y basado en distintos orígenes, desde mandatos religiosos hasta prejuicios sociales" (Estacio, 2012). Por una parte considerar este tipo de violaciones ante el derecho de reproducción y libertad es un sesgo de una sociedad patriarca que radica en la práctica de discriminación. Calva (2015), describe a las madres solteras: "Se le llama madre soltera a la mujer que queda embarazada y decide ser padre y madre a la vez debido a la ausencia paternal del hombre que ayudo a engendrar la semilla de la vida. Las circunstancias por las que una mujer se convierte en madre soltera son variables, desde un enamoramiento fugaz, hasta el hecho de confiar plenamente en el compañero para evitar el embarazo; pero la naturaleza es muy sabia y nosotras a veces imprudentes 
o inocentes o confiadas; hay muchos casos así, donde relatan que pidieron a su pareja que no hubiera eyaculación dentro, y confiaron".

La investigación observó el estigma social desde el aspecto descriptivo, mediante el uso de técnicas e instrumentos que se validaron en las tres fases aplicativas del mismo. Los resultados inciden que el apoyo familiar es una causa directamente sustancial para el desarrollo de una madre soltera, más aún cuando ésta cursa los estudios universitarios, los cuales causan un nivel de estigma frente al nivel de cultura. Los sectores analizados fueron relativamente necesarios para el aporte de investigación.

\section{Método}

El presente estudio estuvo orientado bajo un enfoque positivista, con una metodología cuantitativa, descriptiva, analítica, exploratoria, bibliográfica y estadística. Se resalta ciertas características sociales emergidas en la condición del rol de las madres solteras, quienes se encuentran incorporadas como estudiantes en la Facultad de Ciencias Humanística y Sociales de la Universidad Técnica de Manabí (UTM), de diferentes cantones pertenecientes a la Provincia de Manabí, siendo la población objeto cincuenta (50) estudiantes seleccionadas aleatoriamente.

\section{Desarrollo}

Para Huerta (2019), "en la región de América Latina la figura de cuidado adjudicada a las mujeres está fuertemente asociada con la función materna; por lo tanto, el que ellas se identifiquen con las labores de cuidado las representa como buenas madres, pues el cuidar se reconoce socialmente como una virtud propia de las mujeres". Por una parte, "desde la antigüedad se ha atribuido a la madre el cuidado de los hijos y el hogar; aunque actualmente la mujer sale a trabajar, sigue teniendo a cargo el cuidado de los hijos. Se ha observado que este cambio favorece la independencia de la mujer, pues cada vez son más las mamás que no cuentan con el apoyo de una pareja para encargarse de los gastos de su hogar y la crianza de los hijos" (Aguilar, 2017).

Un aspecto importante dentro del desarrollo, con el pasar del tiempo, ha sido el apoyo que tienen las familias para una mujer que asume el rol de ser madre soltera. 
La familia pasa a ser el primer contacto que un sujeto tiene con la sociedad, siendo el espacio que facilita el aprendizaje y ensayo de comportamientos sociales. Frecuentemente es asimilada por una agrupación de personas compuesta por una madre, un padre y sus hijos, quienes componen un grupo primario óptimo para el desarrollo de una persona (Besa, 2017).

"Las madres solteras establecen a la familia de origen como elemento fundamental para configurar su identidad materna universitaria. La universidad es considerada el medio para lograr una formación profesional que les permita adquirir elementos para integrarse al mercado laboral y, en consecuencia, alcanzar independencia económica y residencial". Por otro lado, "las madres primíparas tardías cuestionan el modelo tradicional de familia, prefieren ser sujetos con capacidad de decidir cuándo y cómo ser madres, y lo ejercen de forma responsable. Deciden serlo cuando reúnen las condiciones necesarias para ello, calculando las consecuencias y las asumen con sus propios medios y recursos" (Coronel, 2017).

Por consiguiente, es de importancia resaltar que la mujer ha sido considerada desde muchas décadas un ser de relevancia en la vida, principalmente por ser productora de vidas al mundo, por supuesto con el apoyo inaudito del hombre con su semental. Unido esto, la excelente representación que la ha marcado como madre trabajadora, amorosa, responsable atenta a todo lo concerniente al compromiso que asume día a día en la crianza de sus hijos y la responsabilidad en todos los ámbitos hacia ellos y en su contexto laboral. En el mismo hilo conductual del presente estudio, se trae a colación lo planteado por el autor Vega (2010), quién expone lo siguiente:

Cuando se habla de la mujer, se habla también de muchos logros que ha tenido durante el transcurso de la historia, sin embargo, es importante señalar que también se ha considerado a esta como un ser estigmatizado, representando algo diferente, lo cual se hace resaltar desde hace mucho tiempo por ejemplo se le ha reprimido en su sexualidad. Principalmente las mujeres que inician a temprana edad su sexualidad, todavía son señaladas socialmente, lo que impide que haya una igualdad de oportunidades, pues muchas de las actividades que pudiesen compartir hombres y mujeres, son truncadas por la misma sociedad. 
Un estado significativo sobre el proceso de responsabilidad en la crianza ha sido una cuestión de sostenibilidad que produce la corresponsabilidad de cada individuo. Por consiguiente, como lo expone Castellanos (2015), "en la actualidad se encuentra que los hombres han asumido la paternidad sin la presencia de una pareja, por diferentes circunstancias como ausencia transitoria, o prolongada de esta, lo que hace que la condición de ser cabeza de familia, no necesariamente sea indispensable el abandono del hogar por parte de su pareja y de las responsabilidades, sino por diferentes factores que hacen que el núcleo familiar este cambiando en la actualidad".

Por otro lado, "las madres solteras que son estudiantes en el sistema de educación superior desarrollan una identidad materna universitaria que les permite establecer modificaciones culturales a través del ejercicio de la capacidad de agencia, por lo que se sirven de redes de apoyo que favorecen el que ingresen y permanezcan en la universidad" (Huerta, 2019). Es importante resaltar la acción que tienen las madres solteras dentro del contexto universitario, la prevalencia de tal objeto precede de las circunstancias en las que se encuentran y de cuál es el precedente de cada una de ellas.

En este contexto, desde hace décadas se puede evidenciar el incremento alto de trayectoria que han tenido las mujeres en la inserción en el ámbito universitario, en víspera de su crecimiento personal-profesional, así como para el bienestar de su familia, y más aún quienes provienen de diversos sectores económicos. Así lo sostienen las autoras Miller y Arvizu (2016) quienes exponen que:

La experiencia cotidiana dentro de los espacios educativos tiene una enorme influencia en las construcciones de los itinerarios biográficos de cada uno de los estudiantes, que involucran tanto la cuestión institucional como la socialización que entablan los sujetos al interior y el exterior de las universidades. Para el caso de la maternidad, se puede concebir que, por una parte, estas mujeres tienen una condición que les lleva a planificar de forma distinta su vida dentro y fuera de los espacios universitarios, pues deben atender responsabilidades con sus estudios y con sus familias. Aunque no es sólo la condición de maternidad la que influye en la manera como las estudiantes generan estrategias y conviven en las instituciones de educación superior, son también las formas en que socializan, la identidad 
que se generan, y la forma de concebir y de entender el espacio institucional, las singularidades que permitirán su permanencia en las universidades, en conjunto con su desenvolvimiento exitoso en otros espacios.

"Legitimar jurídicamente las identidades de las madres solteras es una forma de reconocer su ciudadanía, pues a pesar que su estancia es temporal en la universidad, aun así tienen derechos que son vigentes de inicio al término de su formación profesional, y de cumplirse esta ley permitirá una permanencia más democrática en la educación superior" (Huerta, 2015). Es por eso que en este contexto investigativo es de importancia resaltar lo tipificado en la Ley Orgánica de Educación Superior de Ecuador (2010), en su Título I, Capítulo I, en los Artículos 2 y 4 , que exponen que el objeto de esta Ley garantizar el derecho a la educación superior de calidad y sin discriminación alguna, y que el derecho a la educación superior consiste en el ejercicio efectivo de la igualdad de oportunidades, respectivamente. "Ecuador ha experimentado un sin número de cambios en la política, economía, educación, entre otros. Día a día la población está más preparada para dichos cambios. La presente investigación se basa en los retos que tienen las madres solteras en la vida universitaria estudiantil. Sin embargo, también se realiza enfoques desde el ámbito profesional, familiar y social, para evidenciar el ambiente en que se desenvuelven las madres solteras constando los factores que las pueden afectar o aquellos que pueden propiciar un empuje a la autoestima de la misma" (Barcia, 2017).

Dentro del marco de Ecuador, las madres solteras son la visibilidad del proceso de transferir solitario y el aspecto monoparental, es así que sería un proceso intrínseco dentro de las sociedades contemporáneas de este Estado. Para la autora Ayovi (2018), las madres solteras presentan afectaciones de salud física y psicológica, encontrándose en ellas carencia de revisiones médicas anuales por el déficit de turno o por el tiempo de demora al ser atendidas. Asimismo, la mayoría de ellas no realizan actividades físicas llevando una vida sedentaria. Aun cuando las madres solteras presentan afectaciones físicas y psicológicas, se puede evidenciar la despreocupación por el estado de salud y la mejora de su estilo de vida. 
Por consiguiente, el accionar de las madres solteras puede verse vinculado a los sesgos del estigma y de la categorización de los procesos que involucran la práctica social, los elementos influyentes machistas y los sistemas de interpretación sobre el tipo de rol que se presentan en cada una de ellas.

En efecto, si bien se establece el criar y llevar a cargo la responsabilidad que siempre es llevada por el hombre, según el "modelo tradicional", encasilla a la mujer como parte de un proceso de características no bien vista por la sociedad. Sin embargo se debe desvincular este esquema sobre la estigmatización en las mujeres. "La ambivalencia moral y social que históricamente ha trascendido la adicción en las mujeres, recae en la articulación de un mecanismo de defensa y una reproducción social que se circunscriben a la perpetuación de desigualdades y la consolidación de un determinado sistema de control social, más preocupado por la seguridad que por brindar un estado de salud social" (Osuna, 2013).

El estigmatizar no solo es producente de los hechos que se manifiestan dentro del desarrollo personal de la mujer, sino también son aquellos que se fusionan para realizar un declive social, despotencializando elementos críticos sobre "el estigma" y los "aspectos de configuración social". Es en sí que se produce no adecuado para la realización y el comportamiento del acceso igualitario en la mujer: "La persona responsable de estos hogares es una mujer de 30 a 45 años, aproximadamente, de nacionalidad española y, con problemas de desempleo laboral y trabajos inestables. Tienen problemas de ansiedad y depresión y, la vivienda y alimentación han perdido importancia en su vida: viven en casas inhabilitadas de escasos metros cuadrados, comparten vivienda con otras personas y, para poder pagar el alquiler ahorran en alimentación, eliminando dietas nutritivas" (Santibáñez, 2018).

El estigma social desde el imaginario cultural es una fuerte opresión desde el modelo patriarcal que influye en la asignación de roles y estereotipos para inferir en la decisión reproductiva de la mujer, esto puede causar un despliegue de incidencia social, el cual contrae problemáticas que son netamente retroactivas en las sociedades contemporáneas. 


\section{Resultados}

El proceso de obtención de datos se llevó a cabo a través de la encuesta estructurada, integrada por 10 preguntas. Para este fin, el estudio cumple como aspecto el analizar a nivel social, cultural y familiar a las mujeres que son madres solteras estudiantes universitarias de la Universidad Técnica de Manabí. Se toma como población objeto de estudio a la Facultad de Ciencias Humanísticas y Sociales de las diferentes carreras que la conforman.

\section{Primer momento operativo de la investigación:}

Se ejecutó la encuesta estructurada, la cual estuvo conformada por la muestra de madres solteras dentro de la facultad, los primeros campos en determinar la intervención y el enfoque que se le quiere dar a los antecedes que fueron mencionados en los referentes teóricos. El modelo de intervención social seleccionado es el humanista/ existencial ${ }^{4}$. Esto permitió la intervención de forma científica basándose en componentes que determinan el accionar del ser humano como parte del desarrollo.

\section{Segundo momento operativo de la investigación: Estado situacional, demográfico y variables de edades.}

El estado situacional de las mujeres que fueron encuestadas, es que inciden que las posibilidades de acreditar y pertenecer al sistema de educación superior, gracias a las políticas de inclusión y que existe un mayor aporte desde las aulas universitarias. Las edades de las mujeres seleccionadas para el respectivo estudio oscilan entre 18 - 33 años de edad, pertenecen al espacio sociodemográfico de sectores rurales, como de los cantones: Portoviejo, Santa Ana, Manta, 24 de Mayo, Rocafuerte, Junín.

Tercer momento operativo de la investigación: Resultados investigativos a través del procesamiento de datos estadísticos.

\footnotetext{
4 Frente al conductismo y al psicoanálisis, en Estados Unidos se originó el movimiento de psicología humanista, según el cual la persona busca autor realizarse, siendo éste el propósito general que guía sus acciones y da sentido a su vida. Maslow (1908-1970) denominó a esta tendencia como la «tercera fuerza» (en psicología), señalando así su rechazo a un tiempo del conductismo y del psicoanálisis.
} 
88

\section{Tabla 1}

¿Usted trabaja?

\begin{tabular}{lcc}
\hline Ítems & Cantidad & Porcentaje \\
\hline Si & 18 & 36 \\
No & 32 & 64 \\
\hline Total & 50 & $100 \%$ \\
\hline
\end{tabular}

En la primera tabla se puede observar que apenas el $36 \%$ de las mujeres que fueron encuestadas cumplen con el acceso a un puesto laboral, mientras el $64 \%$ de las mujeres no desarrollan alguna actividad laboral. Lo prescindible en este caso es bosquejar cuáles son los factores por los que ellas no desarrollan algún tipo de actividad económica, quedando a modo de interrogante los factores, el sistema de desarrollo laboral o el perfil que deben cumplir para el acceso a un puesto laboral. Espinoza (2017), indica que en el Ecuador la discriminación por sexo, por tener condición de ser madre soltera, por pertenecer a una determinada etnia o padecer de alguna discapacidad presentaron cifras representativas, además que existe una estructura institucional que protege a sus nacionales contra la discriminación.

\section{Tabla 2}

¿Cómo está constituida su familia?

\begin{tabular}{lcc}
\hline Ítems & Cantidad & Porcentaje \\
\hline Padres e hijos & 12 & 24 \\
Padres, hijos y abuelos & 20 & 40 \\
Madre, abuelos. Hijos & 18 & 36 \\
\hline Total & 50 & $100 \%$ \\
\hline
\end{tabular}

En la tabla 2 se muestra que de las mujeres que fueron encuestadas, el $40 \%$ responden que su familia están constituidas por varios miembros como padres, hijos y abuelos; el $36 \%$ indican que están constituidas por madres, abuelos e hijos. Las categorías que se asignaron para la encuesta revelan que son familias que provienen 
con estructuras de jerarquías y de sistematización dentro de su mismo ambiente social. "Los cambios en la familia durante los últimos 40 años han sido los más profundos y convulsivos de los últimos 20 siglos. La familia tradicional ha cambiado. Aparecen hoy un gran número de modelos que alteran los parámetros con los que se entendía la vida familiar" (Valdivia, 2008).

\section{Tabla 3}

Principal desventaja de ser madre soltera.

\begin{tabular}{lcc}
\hline Ítems & Cantidad & Porcentaje \\
\hline Aspecto económico & 20 & 40 \\
No tener el apoyo de la pareja & 20 & 40 \\
La crítica de la sociedad & 10 & 20 \\
\hline Total & 50 & $100 \%$ \\
\hline
\end{tabular}

Como se aprecia en la tabla 3, el $40 \%$ de las madres solteras encuestadas indican como desventaja el aspecto económico dentro de su desarrollo, esto puede darse en el sentido que las mujeres cargan con el peso de mantener y brindar un desarrollo como parte de su rol, pero las condiciones escasas de trabajo son una de las principales causas por las que se considera desventaja el hecho de ser madre soltera. Un $40 \%$ también incide que la desventaja de no tener la pareja para el desarrollo familiar puede provocar un desequilibrio entre las corresponsabilidades adquiridas en el hogar y en la formación integral del niño o niña. "Indiscutiblemente, el principal aspecto negativo que tiene en contra el afrontar la llegada de un bebé en solitario es que no hay ayuda de ninguna pareja. Eso supone que la mujer tenga que hacerse cargo por sí sola del cuidado del pequeño, del control constante de su bienestar, de las tareas del hogar, de llevar a casa el dinero para poder sobrevivir y de estar a su lado cuando se encuentre enfermo" (OKDIARIO, 2015).

\section{Discusión}

La mujer es uno de los individuos que está relacionado con el hecho de dar vida, su estado es único en el desarrollo y sus esferas de conexión de naturalidad las hacen 
un ser diverso. Por una parte, ante este proceso, es conllevar el contraste con teorías que sean relacionadas con el concebir, y por ello que el ecofeminismo es la consideración de una constelación de iniciativas promovidas por mujeres en todo el mundo que quieren contribuir a asegurar los fundamentos de la vida y garantizar que el bienestar sea para todos los seres vivos que formamos parte de este planeta (Tejero, 2007). Realizando un proceso sobre este tema, la revisión de los resultados descriptivos de la investigación plantea una serie de ideas que son expuestas por las mujeres y las características que afirman ellas como madres solteras: "Tengo el apoyo de mis padres en el cuidado de mi bebé y así puedo ir a estudiar", "Para todo hay tiempo y uno se puede organizar", "A pesar de no contar con el apoyo de mi pareja, tuve el apoyo de mi familia y eso me impulsó a seguir adelante5". Es evidente interpretar que los contextos por los que cruzan las madres solteras son un hecho distinto, y que el desarrollo personal y profesional depende del tipo de apoyo con los que ellas cuentan. Huerta (2018), "reincide en que el concepto de madre soltera como constructo social ha sufrido aparentemente pocas modificaciones en el devenir histórico, a pesar de que se diversificaron las formas en que se definen y convierten las mujeres en madres solteras".

Se puede indicar que el imaginario de las personas está netamente relacionado en que una mujer no puede ejercer voluntad cuando pasa al rol de ser madre soltera y que los estigmas que los persiguen, justamente se ven involucrados en el no poder desarrollar una carrera universitaria. La cultura machista y sexista hace regocijar una carga sobre la responsabilidad y se visualiza que no existe corresponsabilidad, sino un plus ante el poder que aún ejercen ciertos hombres sobre la continuidad machista de la sociedad.

Iniciar con este pronunciamiento como el que expresaron: "Vivimos en una sociedad que no ven con buenos ojos el hecho de ser madre soltera, pero a medida que pasa el tiempo, eso va cambiando, las mujeres podemos salir adelante por nuestro bien y por el de los hijos, aun no teniendo el apoyo de la pareja, lo importante es superarse y demostrar al mundo que si podemos"b. La principal caracterización de este proceso es el autoconocimiento, el empoderamiento y el romper el estigma al que

5 Instrumento aplicado a 50 madres solteras de la facultad de Ciencias Humanísticas y Sociales. 6 Ibid. 
están expuestas las mujeres solteras y que ejercen el rol de madres sin pareja. No obstante, Jocile (2009) incide que el énfasis en romper esa imagen ha producido, desde nuestro punto de vista, un nuevo estereotipo, solo que con rasgos inversos al anterior. Desde las ciencias sociales, los medios de comunicación han difundido la idea de que éstas constituyen un colectivo de mujeres empoderadas, que ocupan una posición social medio-alta con formación universitaria, ingresos elevados, estabilidad laboral, éxito profesional, etc; que se plasma, entre otras cosas, en la capacidad de auto gestionar sus propias vidas y en la autoconciencia de tener competencia suficiente para ello.

Por otro sentido, la tesis titulada "Ser Estudiantes, Madres y Padres: Una dualidad cotidiana", presentada por Castañeda (2015), indica que

La maternidad/paternidad se configura como una doble ruptura en sus trayectorias. El primer nivel corresponde a su vida cotidiana, que los obliga a reorganizar sus prioridades y tiempos para encajar la nueva demanda de responsabilidades. Esta ruptura no es personal. Los cambios afectan también al entorno inmediato del sujeto y en la reestructuración entra en juego el círculo completo de espacios, redes y vínculos a los que el joven está asociado. Es, sin duda, un fenómeno social.

Realizando un despliegue sobre este mismo abordaje es crucial interseccionalizar los pensamientos de las mujeres que formaron parte de estudio las frases sobre el limitante fueron: "A veces no existe el apoyo familiar", "Hay que hacer un esfuerzo para salir adelante" "Se nos hace muy difícil seguir adelante sin el apoyo familiar". Es necesario comprender este contexto sobre cómo se desarrolló este tipo de pensamientos y cuáles son los factores que residen ante la presencia de los casos de las mujeres que están como población estudiantil universitaria. 


\section{2}

\section{Conclusiones}

El estigma social desde el imaginario cultural, focaliza a la madre soltera como un individuo que no cumple los patrones asociados al rol de pertenencia, se establece que la mujer que decide el formar una familia monoparental declive en rolizar la carga de discriminación en las pocas oportunidades que tiene para el acceso laboral, social y educativo. Asimismo, se configura la identidad de la mujer como parte de un sector excluyente que no puede estar dinamizado en situaciones de responsabilidad.

Dentro de los resultados de la investigación, se concluye que las madres solteras afirman que uno de los principales apoyos que tienen es la familia, que está conformada por el papá, mamá, hermanos y en otros casos por abuelos. Esto se debe a que el sistema al que pertenecen es de representación cultural con extensiones de familias numerosas, las cuales proceden con características de crianzas y dentro de su genograma influyen de manera interseccional como apoyo a cada una de ellas.

En otro aspecto, el factor económico es una limitante para las madres solteras porque requieren de la sustitución para acceder a cubrir las necesidades que se presentan dentro de la carga familiar. Como parte de esto, muchas optan por buscar de empleo de medio tiempo, los cuales le pueden favorecer a mejor su calidad de vida y del rol reasignado. Esto es influyente y en las sociedades actuales porque se deben buscar mecanismo de inserción y de apoyos como parte de la red de apoyos en la sororidad y esto es precisamente lo que el feminismo actual busca en parte de mejorar la vida de una madre soltera.

La responsabilidad compartida es un factor de incidencia, el declive del hombre en muchos casos suele darse a la no corresponsabilidad compartida, pero en ciertos casos son las familias el soporte vinculante para evitar esta carga de mejoras en la forma de vida. 


\section{Referencias}

Aguilar, D, Medina, B, Martínez, R. (2017). El sentido de vida en madres solas. Integración Académica en Psicología, 5 (13). -121-131. Recuperado de http://integracion-

academica.org/attachments/article/158/10\%20Madres\%20solas\%20\%20DAguilar\%20BMedina\%20RMartinez.pdf

Ayovi, S. (2018). Factores que influyen en el estilo de vida en la familia monoparental de la ciudadela del buen vivir bendición de dios del cantón Esmeraldas.

(Tesis de pregrado). Pontifica Universidad Católica del Ecuador. Esmeraldas, Ecuador. Recuperado de https://repositorio.pucese.edu.ec/bitstream/123456789/1591/1/AYOV\%C3\%8 D\%20M\%C3\%81RQUEZ\%20SARA\%20RAQUEL.pdf

Asamblea Nacional de la República del Ecuador.(2010). Ley Orgánica de Educación Superior: Recuperado de: https://www.epn.edu.ec/wpcontent/uploads/2018/08/LOES.pdf.

Barcia, M, Medranda, G. (2017): "Los retos de las madres solteras en la formación académica universitaria: caso estudiantes de la escuela de trabajo social de la Universidad Técnica de Manabí", Revista Contribuciones a las Ciencias Sociales, Recuperado de:

http://www.eumed.net/rev/cccss/2017/01/madres.html

Besa, R. (2017). "Efectos del ser madre soltera en una sociedad que regula y norma el quehacer cotidiano". (Tesis de pregrado). Universidad Académica de Humanismo Cristiano. Santiago de Chile, Chile. Recuperado de http://bibliotecadigital.academia.cl/bitstream/handle/123456789/4162/TTRAS O\%20516.pdf? sequence $=1$ \& isAllowed $=y$

Calva, Y. (2015). Madres solteras y su incidencia en el desarrollo de la autonomía e independencia personal de los niños y niñas del centro de educación inicial Pío Jaramillo Alvarado de la ciudad de Loja. Periodo 2014. (Tesis de pregrado). Universidad de Loja. Loja, Ecuador. Recuperado de http://dspace.unl.edu.ec:9001/jspui/bitstream/123456789/16709/1/TESIS.pdf

Castañeda, M. (2015). Ser Estudiantes, Madres y Padres: Una dualidad cotidiana. (Tesis de posgrado). Facultad de ciencias sociales, Universidad de Chile. 
Santiago, Chile. Recuperado de

http://repositorio.uchile.cl/bitstream/handle/2250/135041/Memoria\%20de\%20Titulo\%2

0-\%20Maternidad\%20y\%20Paternidad\%20Universitaria\%20FINAL.pdf? sequence=1

Castellanos, Y, Garzón, M, Sotelo, C. (2015). Factores que influyen en las madres cabeza de familia para acceder a los procesos de formación que proporciona el banco caja social a sus colaboradores. (Tesis de pregrado). Universidad Piloto de Colombia. Bogotá, Colombia. Recuperado de http://polux.unipiloto.edu.co:8080/00002661.pdf

CEPAL. (2020). Igualdad de género y autonomía de las mujeres deben estar en la base del nuevo modelo de desarrollo que requiere con urgencia la región: CEPAL. Recuperado de https://conferenciamujer.cepal.org/14/es/noticias/igualdad-generoautonomia-mujeres-deben-estar-la-base-nuevo-modelo-desarrollo-querequiere

Coronel, C, Chávez, A, González, M, Guisado, M. (2017). Madres solteras por elección. Una realidad creciente y no reciente. ELSEVIER, 48 (8). 502-503. Recuperado de https://www.elsevier.es/es-revista-atencion-primaria-27-articulo-madressolteras-por-eleccion-una-S0212656717300604

Espinoza, M, Gallegos, D. (2017). Discriminación laboral en Ecuador. Espacios, 39 (23). 1-15. Recuperado de https://www.revistaespacios.com/a18v39n23/a18v39n23p32.pdf

Estacio, V, Medina, O. (2012). Análisis del número de madres solteras en la facultad de comunicación social de la universidad de Guayaquil para la creación de un programa radial de información de ofertas laborales para ellas y orientación familiar. (Tesis de pregrado), Universidad de Guayaquil, Guayaquil, Ecuador. Recuperado de http://repositorio.ug.edu.ec/bitstream/redug/2447/1/tesis\%20proyecto.pdf

Huerta, R. (2015). "Joven, mamá y estudiante: Identidad materna universitaria de "la madre soltera' ". (Tesis doctoral). El colegio de San Luis. Recuperado de https://biblio.colsan.edu.mx/tesis/HuertaMataRosaMaria.pdf 
Huerta, R. (2017). Las madres solteras universitarias: redes de apoyo social e identidad materna. Interscticiones Sociales. Recuperado de http://www.intersticiossociales.com/index.php/is/article/view/199/434

Huerta, R. (2018). Construcción Conceptual de las "Madres Solteras" en México. Revista punto de género, 10. 60-82.

Huerta, R. (2018). El cuerpo de las jóvenes madres solteras universitarias: representaciones y estrategias. La ventana. Revista de estudios de género, 5(47), 207-250. Recuperado de http://www.scielo.org.mx/scielo.php?script=sci_arttext\&pid=s 1405 94362018000100207\&lng=es\&tlng=es

Huerta, R. (2019). Las madres solteras universitarias: redes de apoyo social e identidad materna. Interticisios sociales, 17. 204-231. Recuperado de http://www.scielo.org.mx/pdf/ins/n17/2007-4964-ins-17-203.pdf

Jociles, $M^{a}$, Rivas, A. (2009). Entre el empoderamiento y la vulnerabilidad: la monoparentalidad como proyecto familiar de las MSPE por reproducción asistida y adopción internacional. Revista de Antropología Social, 18. 127-170. Miller, Dinorah; Arvizu, Vanessa. (2016). Ser madre y estudiante. Una exploración de las características de las universitarias con hijos y breves notas para su estudio. Revista de la Educación Superior. México. XLV (1), 177. pp. 17-42.

OKDIARIO. (18 de diciembre del 2015). Desventajas de ser madre soltera. [Mensaje de un blog]. Recuperado de https://okdiario.com/bebes/desventajas-sermadre-soltera-2682113

Osuna, M. (2013). Estigma social en madres puertorriqueñas usuarias de heroína:

Una exploración de las voces femeninas y su entorno social. Revista Puertorriqueña de Psicología, 24. 1-17. Recuperado de http://pepsic.bvsalud.org/pdf/reps/v24/a05.pdf

Santibáñez, R, Flores, N. (2018). Familia monoparental y riesgo de exclusión social. Igual. Revista de género e igualdad, 1. 123-144. doi: I. http://dx.doi.org/10.6018/iQual.307701

Tejero, $M^{a}$. (2007). Mujeres y madres solteras, ecofeminismo e interculturalidad. Medicina Naturista, 1 (1). 2-8. 


\section{6}

Valdivia, C. (2008). La familia: concepto, cambios y nuevo modelo. La Revue de REDIF, (1). 15-22. Recuperado de

https://mimateriaenlinea.unid.edu.mx/dts_cursos_mdl/lic/DE/PF/AM/05/camb ios.pdf

Vega, F. (2010). Situación de las madres solteras, visto desde la teoría de "Erving Goffman". (Tesis de pregrado). Universidad Autónoma del Estado de Hidalgo. Recuperado de https://www.uaeh.edu.mx/nuestro_alumnado/esc_sup/actopan/licenciatura /Situacion\%20de\%20las\%20madres\%20solteras.pdf 